\title{
Routine cine-CMR for assessment of prosthesis- associated mitral regurgitation - a multicenter, multivendor study
}

\author{
Lauren A Simprini ${ }^{*}$, Anika Afroz ${ }^{1}$, Igor Klem³ ${ }^{3}$ Christoph J Jensen ${ }^{3}$, Raymond J Kim³ ${ }^{3}$, John F Heitner ${ }^{4}$, \\ Michael Sood ${ }^{4}$, Elizabeth Chandy ${ }^{4}$, Dipan J Shah ${ }^{5,1}$, Juan C Lopez-Mattei ${ }^{5}$, Robert W Biederman ${ }^{6,7}$, Anthon Fuisz ${ }^{8}$, \\ Kambiz Ghafourian ${ }^{8}$, Jonathan W Weinsaft ${ }^{1,2}$
}

From 16th Annual SCMR Scientific Sessions

San Francisco, CA, USA. 31 January - 3 February 2013

\section{Background}

Mitral regurgitation (MR) is clinically important for patients with prosthetic mitral valves (PMV). While CMR can quantify MR based on flow, this requires dedicated imaging. Cine-CMR (SSFP) provides an alternative approach, whereby MR can be graded based on regurgitation-associated intervoxel dephasing. As cine-CMR is a standard component of nearly all exams, it could be used to screen for patients who warrant further quantitative imaging. Diagnostic performance of cine-CMR for prosthesis-associated MR has not been evaluated.

\section{Methods}

Databases at 6 sites were queried for all patients with PMV in whom CMR and echocardiography were performed within 10 days. Cine-CMR images were retrieved and interpreted using a uniform protocol: $\mathrm{MR}$ was visually graded based solely on jet size (mild $<1 / 3$, moderate $1 / 3-2 / 3$, severe $>2 / 3$ ) in relation to the left atrium. MR was graded in each long axis plane (2-,3-, 4-chamber), with overall severity based on mean grade. Additional parameters included jet directionality, signal intensity (3-grade scale), and pulmonary vein flow reversal. Echocardiography (TTE, TEE) was used as a comparator for MR based on clinically reported data. Cine-CMR was interpreted blinded to patient history and TTE/TEE.

\section{Results}

56 patients with PMV (70\% mechanical, 30\% bio) underwent cine-CMR and echo (TTE 70\%, TEE 48\%) within $2.5 \pm 2.6$ days. Cine-CMR (1.5T, typical $\mathrm{TR}=3 \mathrm{msec}$, $\mathrm{TE}=1 \mathrm{msec}, \mathrm{BW}=977 \mathrm{~Hz} /$ pixel) was performed using commercial scanners (Siemens 59\%/GE 36\%/Philips 5\%). MR was present on cine-CMR in $77 \%$ of patients (mild $43 \%$, moderate $14 \%$, severe $20 \%$ ), and varied in direction (central 88\%, eccentric 12\%). Patients with severe MR had higher prevalence of dense regurgitant jets $(73 \%$ vs. $3 \%, \mathrm{p}<0.001)$, more frequent pulmonary vein reversal $(55 \%$ vs. $3 \%, \mathrm{p}<0.001)$, and larger left atria $(5.7 \pm 1.0 \mathrm{~cm}$ vs. $4.7 \pm 1.4 \mathrm{~cm}, \mathrm{p}=0.03)$ than did those with lesser $\mathrm{MR}$, but did not differ based on LVEF $(53 \pm 14 \%$ vs. $49 \pm 15 \%$, $\mathrm{p}=0.4$ ). Compared to TEE, cine-CMR yielded excellent diagnostic accuracy (96\%) for severe MR (Table); accuracy was also high (93\%) when a broader TEE threshold ( $\geq$ moderate MR) was applied. Among patients with TTE

Table 1 Cine-CMR diagnostic performance for prosthesis-associated mitral regurgitation

\begin{tabular}{cccccc}
\hline & Sensitivity & Specificity & Accuracy & Positive predictive value & Negative predictive value \\
\hline Severe MR & $100 \%(6 / 6)$ & $95 \%(20 / 21)$ & $96 \%(26 / 27)$ & $86 \%(6 / 7)$ & $100 \%(20 / 20)$ \\
\hline Substantial ( $\geq$ moderate) MR & $100 \%(9 / 9)$ & $89 \%(16 / 18)$ & $93 \%(25 / 27)$ & $82 \%(9 / 11)$ & $100 \%(16 / 16)$ \\
\hline
\end{tabular}

Calculations based on transesophageal echocardiography reference standard.

\footnotetext{
${ }^{2}$ Medicine/Cardiology, Memorial Sloan Kettering Cancer Center, New York,
} NY, USA 
and cine-CMR, no patients had severe MR on TTE missed by cine-CMR, whereas TTE was discordant (lesser MR) in 83\% (5/6) with severe MR on cine-CMR. Among those with all three tests $(\mathrm{n}=10)$, TEE-evidenced severe MR was diagnosed by TTE in $50 \%(1 / 2)$, whereas cine-CMR diagnosed severe MR in all (2/2) cases.

\section{Conclusions}

Among a blinded multicenter cohort with PMV, cine-CMR yielded excellent diagnostic performance (accuracy $=96 \%$ ) for TEE-evidenced severe MR. Findings support use of cine-CMR for non-invasive assessment of prosthesis-associated MR.

\section{Funding}

K23 HL 102249-01.

\section{Author details}

${ }^{1}$ Medicine/Cardiology, Weill Cornell Medical College, New York, NY, USA. ${ }^{2}$ Medicine/Cardiology, Memorial Sloan Kettering Cancer Center, New York, NY, USA. ${ }^{3}$ Duke Cardiovascular Magnetic Resonance Center, Durham, NC, USA. ${ }^{4}$ New York Methodist Hospital, Brooklyn, NY, USA. ${ }^{5}$ The Methodist DeBakey Heart \& Vascular Center, Houston, TX, USA. ${ }^{6}$ Allegheny General Hospital, Pittsburg, PA, USA. 'Drexel University College of Medicine,

Philadelphia, PA, USA. ${ }^{8}$ Washington Hospital Center, Washington, DC, USA.

Published: 30 January 2013

doi:10.1186/1532-429X-15-S1-E100

Cite this article as: Simprini et al: Routine cine-CMR for assessment of prosthesis-associated mitral regurgitation - a multicenter, multivendor study. Journal of Cardiovascular Magnetic Resonance 2013 15(Suppl 1):E100.
Submit your next manuscript to BioMed Central and take full advantage of:

- Convenient online submission

- Thorough peer review

- No space constraints or color figure charges

- Immediate publication on acceptance

- Inclusion in PubMed, CAS, Scopus and Google Scholar

- Research which is freely available for redistribution

Submit your manuscript at www.biomedcentral.com/submit
C Biomed Central 